\title{
KLF4 regulates adult lung tumor-initiating cells and represses K-Ras-mediated lung cancer
}

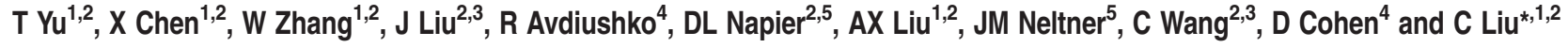

Lung cancer is the leading cause of cancer-related mortality in both men and women worldwide. To identify novel factors that contribute to lung cancer pathogenesis, we analyzed a lung cancer database from The Cancer Genome Atlas and found that Krüppel-like Factor 4 (KLF4) expression is significantly lower in patients' lung cancer tissue than in normal lung tissue. In addition, we identified seven missense mutations in the KLF4 gene. KLF4 is a transcription factor that regulates cell proliferation and differentiation as well as the self-renewal of stem cells. To understand the role of KLF4 in the lung, we generated a tamoxifeninduced KIf4 knockout mouse model. We found that KLF4 inhibits lung cancer cell growth and that depletion of KIf4 altered the differentiation pattern in the developing lung. To understand how KLF4 functions during lung tumorigenesis, we generated the $K$-ras ${ }^{L S L-G 12 D /+} ; K I f 4^{f l / f I}$ mouse model, and we used adenovirus-expressed Cre to induce $K$-ras activation and KIf4 depletion in the lung. Although KIf4 deletion alone or K-ras mutation alone can trigger lung tumor formation, Klf4 deletion combined with K-ras mutation significantly enhanced lung tumor formation. We also found that KIf4 deletion in conjunction with $K$-ras activation caused lung inflammation. To understand the mechanism whereby KLF4 is regulated during lung tumorigenesis, we analyzed KLF4 promoter methylation and the profiles of epigenetic factors. We found that Class I histone deacetylases (HDACs) are overexpressed in lung cancer and that HDAC inhibitors induced expression of KLF4 and inhibited proliferation of lung cancer cells, suggesting that KLF4 is probably repressed by histone acetylation and that HDACs are valuable drug targets for lung cancer treatment.

Cell Death and Differentiation (2016) 23, 207-215; doi:10.1038/cdd.2015.85; published online 26 June 2015

Lung cancer is the most commonly diagnosed cancer among men and women and the leading cause of cancer deaths in the United States. ${ }^{1}$ Mutations in multiple genetic pathways, including EGFR, TP53 and K-RAS, are linked to lung tumorigenesis. KRAS mutations are limited to non-small-cell lung cancer, which is the most prevalent form of lung cancer. ${ }^{2}$ The K-ras protein belongs to a small GTP-binding protein family, which acquires transforming activity with specific mutations, such as a mutation at codon 12. ${ }^{3,4}$ These mutations result in a decrease in GTPase activity and constitutive activation of the K-ras signaling, which actively promotes proliferation and cell survival and ultimately leads to cancer. KRAS mutations are found in more than one-third of human lung adenocarcinomas. Somatic activation of the K-ras gene causes early-onset lung cancer in mice. ${ }^{4}$

Krüppel-like Factor 4 (KLF4) was initially identified as a zinc finger transcription factor enriched in the epithelium of intestine and skin. ${ }^{5,6}$ Later, it was found in a variety of other tissues, such as thymus, cornea, cardiac myocytes and lymphocytes. ${ }^{7-10}$ KLF4 has an important role in the development and cell differentiation. ${ }^{6,11,12}$ In normal lung tissue, KLF4 is expressed in fibroblasts and airway epithelial cells, and was found to be the most significantly altered lung gene at birth. ${ }^{13}$ KLF4 is downregulated in gastrointestinal cancers and has been identified as a tumor suppressor in many types of cancer. ${ }^{14-16}$ As one of the four factors that induce pluripotent stem cells, KLF4 has a role in cell fate reprogramming and selfrenewal of embryonic stem cells. ${ }^{17,18}$ In vitro studies indicated that KLF4 inhibits the growth of many cancer cell lines, including lung cancer cells. ${ }^{19}$ However, the function and regulation of KLF4 in vivo in the lung and lung cancer are still not known.

The cell linage in the lung has been well studied. The major component of the trachea and the proximal conducting airways are basal secretary ciliated cells; bronchiolar and terminal bronchiolar epithelium are mainly composed of nonciliated columnar Clara cells, and alveolar epithelium are composed of type I and type II cells (named AT1 and AT2 cells, respectively). After birth, AT2 cells functions as stem cells in the lung. ${ }^{20,21}$ The self-renewal of AT2 cells is regulated by EGFR and K-ras signals. ${ }^{21}$ In patients, lung adenocarcinoma often stains positive for Clara cell marker CC10 (Clara cell antigen 10) and AT2 cell marker SP-C (surfactant protein C), which led to the hypothesis that Clara cells and AT2 cells might be cells of origin of lung adenocarcinoma. In a recent study, $K$-ras was activated by polr2a-Cre ${ }^{\mathrm{ER}}$, which led to tumors arising only in the lung. Although Cre recombination was detected in the alveoli, bronchioles and the bronchioalveolar

\footnotetext{
${ }^{1}$ Department of Molecular and Cellular Biochemistry, University of Kentucky, Lexington, KY 40506-0509, USA; ${ }^{2}$ Markey Cancer Center, University of Kentucky, Lexington, KY 40506-0509, USA; ${ }^{3}$ Department of Biostatistics, University of Kentucky, Lexington, KY 40506-0509, USA; ${ }^{4}$ Department of Microbiology, Immunology and Molecular Genetics, University of Kentucky, Lexington, KY 40506-0509, USA and ${ }^{5}$ Department of Pathology, University of Kentucky, Lexington, KY 40506-0509, USA

${ }^{*}$ Corresponding author: C Liu, Markey Cancer Center, Department of Molecular and Cellular Biochemistry, University of Kentucky, 741 S. Limestone, B375 BBSRB, Lexington, KY 40506-0509, USA. Tel: 859323 4558; Fax: 859257 6030; E-mail: chunming.liu@uky.edu

Abbreviations: TCGA, The Cancer Genome Atlas; KLF4, Krüppel-like Factor 4; HDAC, histone deacetylases; SP-C, surfactant protein C; CC10, Clara cell antigen 10; BADJ, bronchioalveolar duct junction; IHC, immunohistochemistry; H\&E, hematoxylin and eosin; NaBt, sodium butyrate; MPO, Myeloperoxidase Received 16.2.15; revised 29.4.15; accepted 22.5.15; Edited by S Fulda; published online 26.6.15
} 
duct junction (BADJ), only alveolar lesions progressed to more advanced adenomas and adenocarcinomas, which were composed exclusively of SP-C ${ }^{+}$cells. $^{22}$ In another study, $K$-ras was activated in either AT2 cells or Clara cells by adenovirus-expressed SP-C-Cre or CC10-Cre. Both AT2 cells and Clara cells had the ability to initiate malignant transformation, but the initiating cell type influences the type of tumors that arose. ${ }^{23}$ These findings suggest that AT2 cells are the major origin of lung tumorigenesis.

In this study, we analyzed the function of KLF4 in lung homeostasis and lung cancer using novel mouse models. We also examined the mechanisms of KLF4 regulation in lung cancer.

\section{Results}

KIf4 is a putative tumor suppressor for lung cancer. KLF4 has been identified as a tumor suppressor for gastrointestinal cancers but was also suggested as an oncogene for breast cancer. ${ }^{24,25}$ KLF4 mutations have been identified in colon cancer and meningiomas. ${ }^{14,26,27}$ To examine the function of KLF4 in lung cancer, we analyzed KLF4 mutations in lung cancer patients from the The Cancer Genome Atlas (TCGA) adenocarcinoma database. In all, 9 out of 488 patients had KLF4 mutations. Seven out of these nine mutations were found in the activation domain and the three zinc finger regions of the KLF4 gene (Figure 1a). One

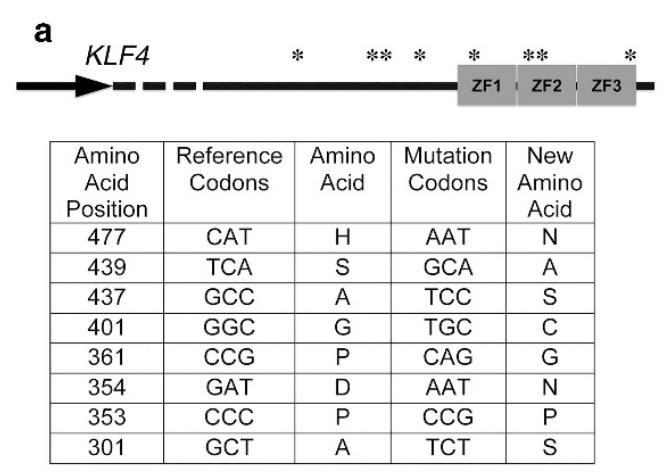

b

c

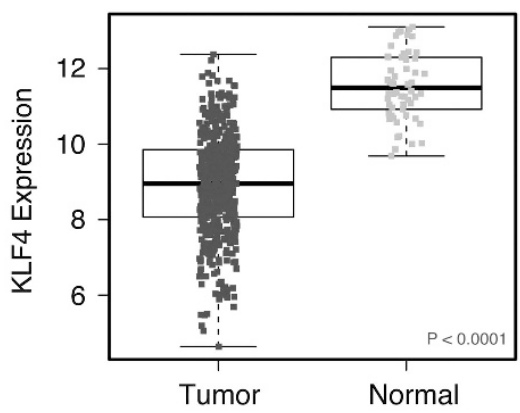

$\mathrm{H} 460$
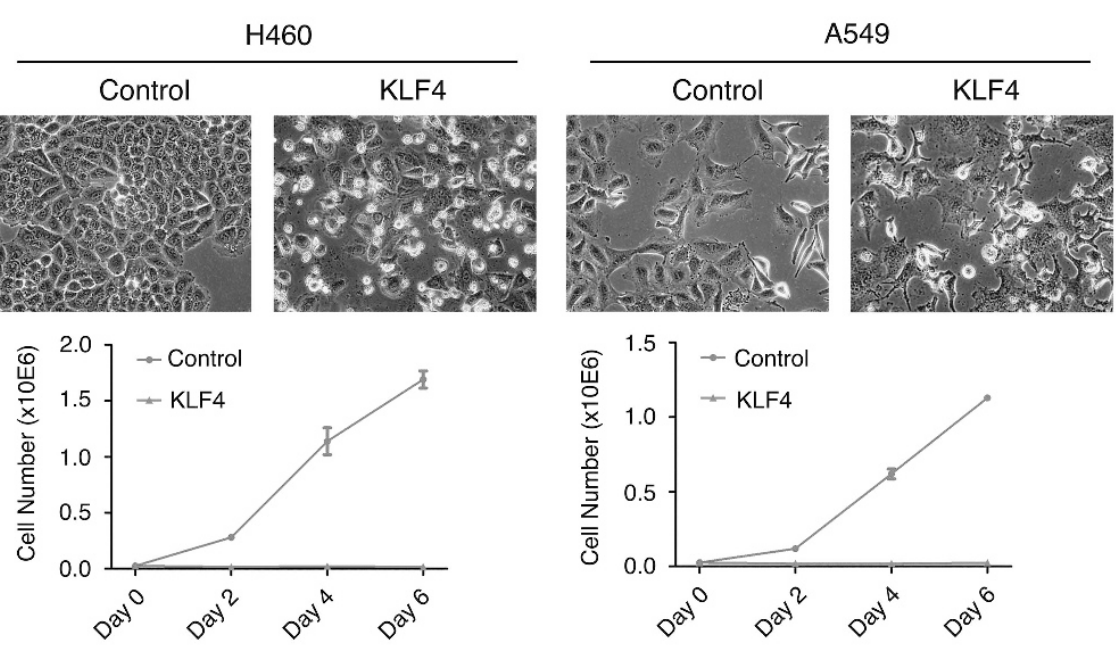

d

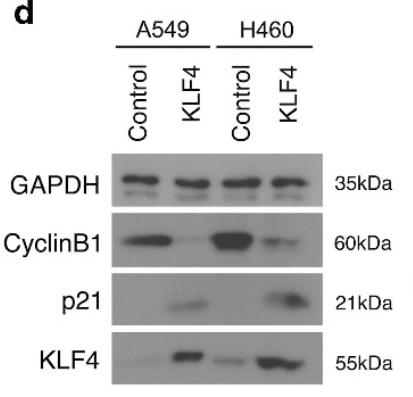

e

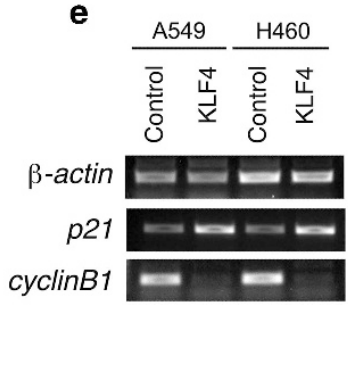

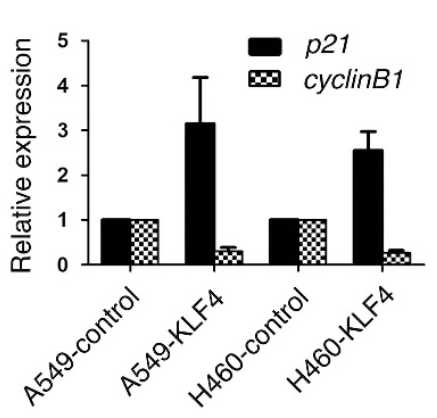

Figure 1 Klf4 is a putative tumor suppressor for lung cancer. (a) Schematic diagram and table list of mutational sites in the KLF4 gene domains. ZF, zinc finger domain; "*', Mutational sites, details of which are explained in the table below. (b) KLF4 expression in normal and lung tumor tissues. The $P$ value was calculated based on a linear mixed model. (c) Top: Morphology of lung cancer cells with ectopic KLF4 expression. Middle and bottom: growth curve of lung cancer cell lines A549 and H460. 'Control', vector-virusinfected cells. (d and e) Western blot (d), semi-quantitative RT-PCR and quantification (e) of expression of KLF4 target genes in lung cancer cell lines with the effect of overexpression of KLF4. Data are represented as mean \pm S.D 
mutation was found in the intron and one was a silent mutation. We also analyzed the small-cell lung carcinoma data from TCGA and found only one case with KLF4 mutation (Supplementary Figure 1a).

KLF4 has the ability to repress cyclinB1 Luciferase reporter. ${ }^{15,28}$ To examine the relationship between mutations and functions of KLF4, we made seven KLF4 constructs containing each of the nine point mutations or containing two point mutations that are close together, and we tested their functions in cyclinB1 reporter assay. These mutations have no significant effect on KLF4 function in repressing cyclinB1 promoter activity (Supplementary Figure 1b).

To further examine the potential deregulation of KLF4 in lung cancer, we analyzed the expression level of KLF4 mRNA in human normal lung versus lung tumor tissues from the TCGA adenocarcinoma database and found that KLF4 expression was significantly lower in tumor tissues (Figure 1b, $P<0.0001$ ). Analysis from the small-cell carcinoma database also indicated significantly lower level of KLF4 mRNA expression (Supplementary Figure 1c).

Other members of KLF family transcription factors may also be altered in lung cancers. ${ }^{29}$ To evaluate the correlation of other KLFs in TCGA lung adenocarcinoma, we analyzed mRNA expression level of KLF1-17 in adenocarcinoma patients and found that in addition to KLF4, KLF1, 2, 6, 8, 9, $10,11,12,13,14,15$ and 17 were also significantly lower in tumor compared with normal tissues (Supplementary Figure 2).

To test the role of KLF4 in lung cancer cells, we ectopically expressed KLF4 by lentivirus-mediated delivering in the human lung cancer cell lines $\mathrm{H} 460$ and A549, respectively, and found that $K L F 4$ overexpression inhibited cell growth to a large extent (Figure 1c), which is consistent with the role of KLF4 as a tumor suppressor for lung cancer. We also infected the BEAS-2B (lung epithelial) cell line with KLF4-carrying lentivirus and tested the cell growth rate. We found that KLF4 overexpression had no significant effect on proliferation of these cells, probably because of the endogenous KLF4 expression (Supplementary Figure 3).

To test the molecular effects of KLF4 overexpression in the lung cancer cell lines, we analyzed the protein expression of KLF4 and target genes by western blot (Figure 1d), and tested mRNA expression of KLF4 target genes by reverse transcriptase PCR (RT-PCR; Figure 1e). We found that $p 21^{C i p 1 / W A F}$ was upregulated, whereas cyclin B1 was downregulated $48 \mathrm{~h}$ post transfection (Figures $1 \mathrm{~d}$ and $\mathrm{e}$ ), suggesting that KLF4 represses lung cancer cell growth by regulating cell cycle and cell proliferation. Deregulation of KLF4 could increase cell proliferation during lung tumorigenesis.

KIf4 ablation altered the differentiation pattern in mouse lung. To test the function of KLF4 in mouse lung, we generated the Rosa-Cre $; \mathrm{KIf4}^{f / f l}$ mice (Figure 2a), in which Rosa-Cre can be activated by tamoxifen. KLF4 is expressed in the epithelial cells of bronchioles as well as alveolar cells (Figure 2b). Rosa26 locus is ubiquitously expressed in mouse embryos ${ }^{30}$ and thus can actively drive tamoxifen-induced Klf4 deletion in most cells in mouse lung (Figures $2 b-d$ ). Two types of cells have been suggested as the origins of lung cancer: AT2 cells and Clara cells. ${ }^{21-23,31,32}$ a

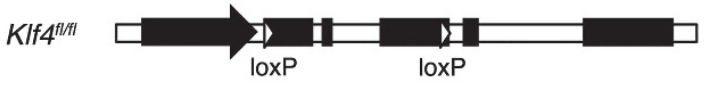

Rosa-CreER ${ }^{\text {T2 }}$ Gt(ROSA26) CreER $^{\text {T2 }}$
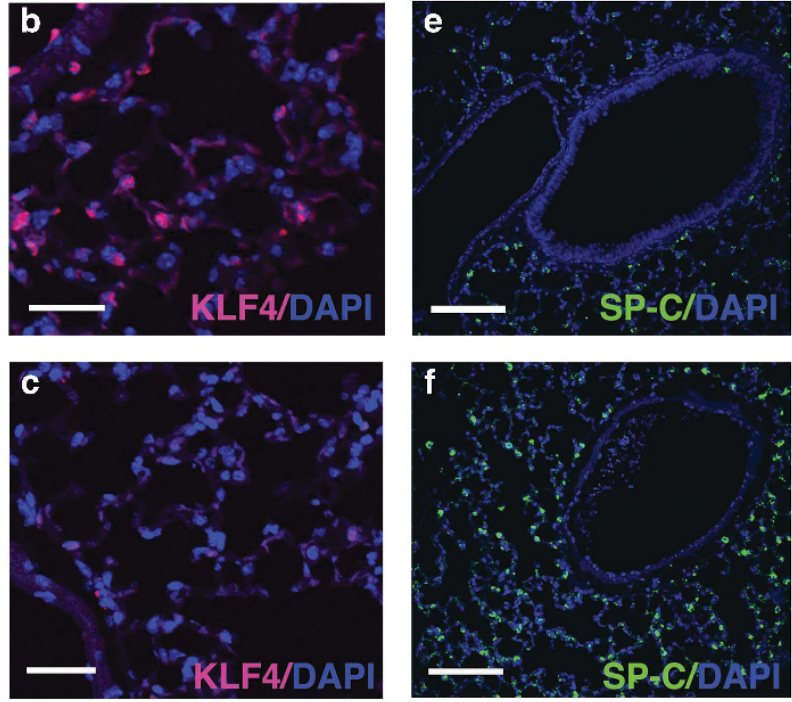

d

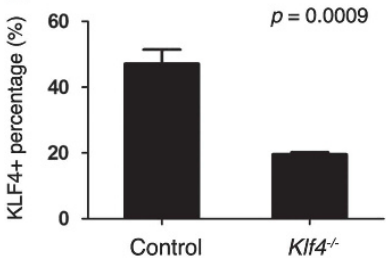

g

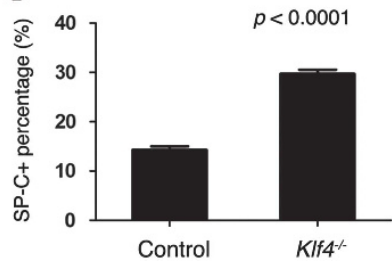

Figure 2 Klf4 ablation altered the differentiation pattern in mouse lung (a) Genetic model of the Rosa-Cre ${ }^{+} ; K_{1 f 4^{f / f t}}$ mouse. LoxP elements allow Cre-induced KIf4 deletion (top); and Rosa26 drives CreER ${ }^{\mathrm{T} 2}$ expression in mouse lung (bottom). (b-d) Immunofluorescent staining for KLF4 (red) in wild-type mouse lung (b) and in Klf4-deleted mouse lung (c). (d) Statistical analysis of KLF4-positive cells in mouse lung. Data are represented as mean \pm S.D. (e-g) Immunofluorescent staining for SP$\mathrm{C}$ in wild-type (e) and Klf4-deleted (f) mouse lung. (g) Statistical analysis of SP-Cpositive cells in mouse lung. Data are represented as mean \pm S.D. Scale bars for (b and c): $30 \mu \mathrm{m}$. Scale bars for (e and f): $100 \mu \mathrm{m}$

SP-C is a marker for AT2 cells and CC10 is a marker for Clara cells. To detect the effects of Klf4 deletion on AT2 cells in the lung, immunofluorescent staining was performed using SP-C antibody and we found that SP-C protein was increased in Klf4-deleted mouse lung tissue (Figures $2 \mathrm{e}-\mathrm{g}$ ). This result suggests that KLF4 has a role inhibiting differentiation of AT2 cell lineage and/or controlling the number of differentiated AT2 cells. To test the effect of KIf4 deletion on other cell types, we stained the lung tissues with antibodies to AT1 cell marker Caveolin 1 (Cav1; Supplementary Figures 4a and b), Clara cell marker CC10 (Supplementary Figures 4c and d), as well as ciliated cell marker FoxJ1 (Supplementary Figures $4 \mathrm{e}$ and $\mathrm{f}$ ). There was no significant difference in these cell markers. AT2 cells have been suggested as the origin of solid tumors in the lung. ${ }^{33}$ Although bronchioalveolar stem cells $\left(\mathrm{CC}_{10} \mathrm{SP}^{+} \mathrm{C}^{+}\right)$were identified as the putative cells of origin of lung adenocarcinoma, ${ }^{32}$ more studies performed recently 
reported that AT2 cells are the predominant cells of origin of adenocarcinoma that is driven by $K$-ras activation. ${ }^{21-23,31,34}$ Our results suggest that KIf4 deletion could be a driving force of lung tumorigenesis through stimulating AT2 cells in mouse lung.

KIf4 deletion facilitates lung tumor formation and progression. To study the role of KLF4 in mouse lung tumorigenesis, we established another mouse model by crossing the KIf4 $4^{f / f l}$ line with the K-ras ${ }^{L S L-G 12 D /+}$ line. In this model, activation of $K$-ras ${ }^{G 12 D}$ can occur simultaneously with Klf4 deletion in the lung. K-RAS mutations are found in more than one-third of human lung adenocarcinomas. Somatic activation of the K-ras gene causes early-onset lung cancer in mice. ${ }^{4}$ Adenovirus-expressed-Cre recombinase was delivered to KIff ${ }^{f / f l}, K$-ras ${ }^{L S L-G 12 D /+}$ or K-ras ${ }^{L S L-G 12 D /+}$; $K l f 4^{f l / f l}$ mouse models by nasal administration, to induce Klf4 deletion, K-ras activation or both in mouse lung (Figure $3 a$ ). As indicated by hematoxylin and eosin (H\&E) staining, tumor tissue appeared in K-ras ${ }^{L S L-G 12 D /+} ; K / f 4^{f l / f l}$ mice as soon as 8 weeks after Cre-induction. Immunofluorescent staining

a
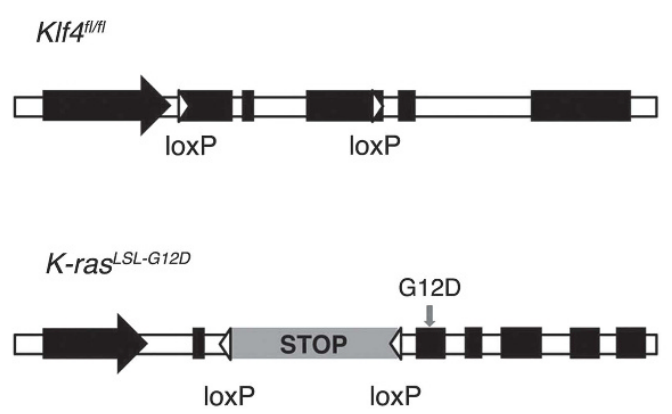

b
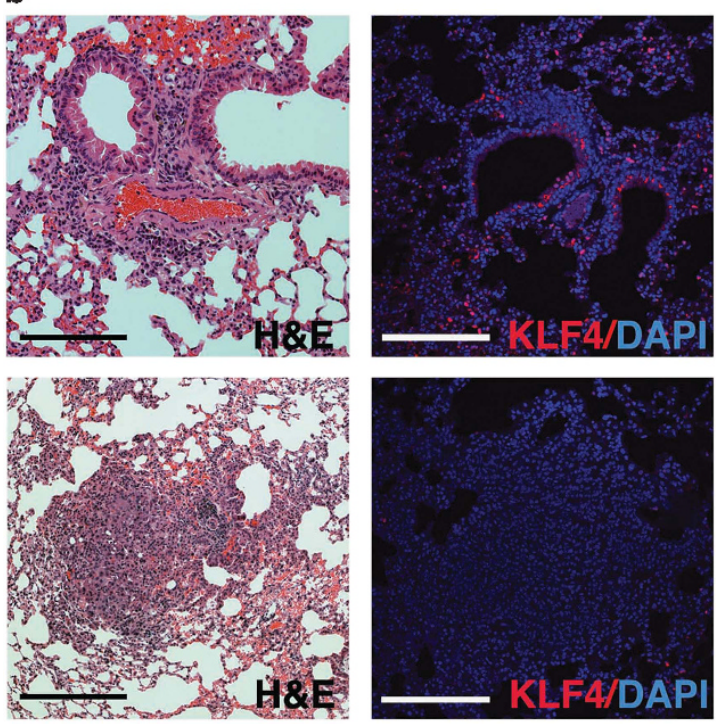

Figure 3 Establishing the genetic model of the $K-$ ras $^{L S L-G 12 D /+} ; K / f 4^{f / / f l}$ mouse. (a) LoxP elements allow Cre-induced Klf4 deletion or G12D mutation-mediated K-ras activation. (b) H\&E staining and KLF4 immunofluorescent staining in normal tissue from wild-type mice (top) and in tumor tissue from $K$-ras ${ }^{L S L-G 12 D /+} ; K / H_{4}^{t / f / I}$ mice (bottom) tissue in the lung, indicating loss of Klf4 expression in lung tumor. Scale bars: $100 \mu \mathrm{m}$ indicated that KIf4 was depleted in these tumors (Figure 3b), suggesting that loss of tumor-suppressing function of KLF4 contributed to tumor formation in these mice.

To test the effect of loss of function of KLF4, along with the effect of $K$-ras ${ }^{G 12 D}$ mutation in lung tumorigenesis, we infected the mouse models (control, KIf $4^{f / f l}, K$-ras ${ }^{L S L-G 12 D /+}$ or $K$-ras ${ }^{L S L-G 12 D /+} ; K I f 4^{f l / f l}$ mice) with Cre-adenovirus and tested the tumor formation in the lungs of these mice. By 8 weeks post infection, diffuse hyperplasia and a few isolated lesions were observed in K-ras ${ }^{L S L-G 12 D /+}$ mouse lung, whereas more hyperplasia and adenoma were observed in K-ras ${ }^{L S L-G 12 D /+}$; $K I f 4^{f l / f l}$ mice (Figures $4 \mathrm{a}$ and $\mathrm{c}$ ). Both immunohistochemistry (IHC) staining and immunofluorescent staining show that SP-C-positive AT2 cells were increased in lung tumor tissue from K-ras ${ }^{L S L-G 12 D /+} ; K I f 4^{f l / f l}$ mice, compared with normal tissue in wild-type mice (Figure 4b), consistent with the $\mathrm{Rosa} \mathrm{Cre}^{+} ; \mathrm{Klf4}^{\text {fl/fl }}$ model in Figures $2 \mathrm{e}-\mathrm{g}$. These results suggest that KIf4 deletion synergized with $K$-ras activation to facilitate tumor formation in the lung. Our findings are consistent with the previous finding that Klf4 deletion sufficiently initiated carcinoma development upon K-ras activation in mouse tongue. ${ }^{35}$

Multiple mechanisms of KLF4 downregulation in lung cancer. KLF4 mutations were identified in fewer than $2 \%$ of the total patients (Figure 1a), suggesting that genetic mutation is not the main cause of KLF4 downregulation in lung cancer. We also analyzed DNA methylation on the KLF4 gene in lung adenocarcinoma patients from the TCGA database. Although a small portion of lung cancer tissues contains KLF4 promoter methylation, there was no significant correlation between $K L F 4$ expression and methylation in lung tumor tissues (Figure 5a, $P=0.8$ ). As KLF4 levels were significantly reduced in lung cancer tissues (Figure 1b), there must be other major factors that repress KLF4 expression during lung tumorigenesis.

Histone modifications bear important roles in regulating cell proliferation and tumor growth. We have reported that KLF4 interacts with histone acetyltransferase and regulates histone acetylation. ${ }^{28,36}$ KLF4 also interacts with histone deacetylases (HDACs) and regulates gene expression. ${ }^{37}$ To identify the major mechanisms of how KLF4 is regulated in the process of lung tumorigeneses, we analyzed the profile of the lung cancer database from TCGA and studied the expression levels of HDACs in both normal and lung tumor tissues. Among HDACs 1-11, expression levels of HDACs 1, 2, 3 and 8, which belong to Class I HDACs, were increased in lung tumor tissues compared with normal lung tissues $(P<0.0001)$, whereas expression of HDACs 4, 5 and 7, which belong to Class Ila HDACs, were decreased (Figure 5b, and Supplementary Figure $5 \mathrm{~b}, P<0.0001)$. These findings are consistent with the previous observation that Class I and Class II HDACs are differentially expressed in cancers, and that the Class Ila HDACs exert a dual role in cancer. ${ }^{38}$

To test the hypothesis that HDACs inhibit KLF4 expression in lung cancer, we treated the lung cancer cell A549 as well as HEK293T cells with HDAC inhibitors and analyzed the expression of KLF4 and its target genes by RT-PCR and real-time PCR. We found that KLF4 was induced in cells treated with several HDAC inhibitors, including suberoylanilide 
a
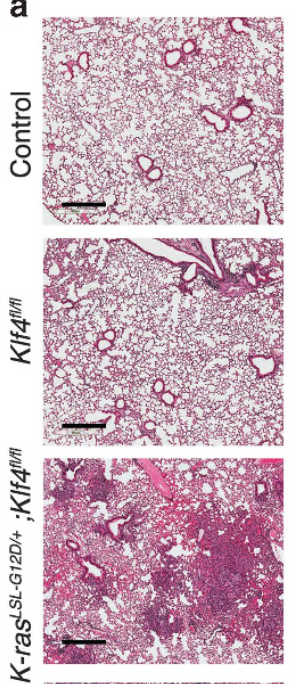

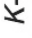

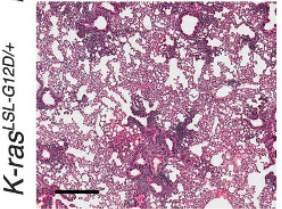

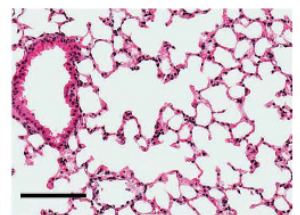
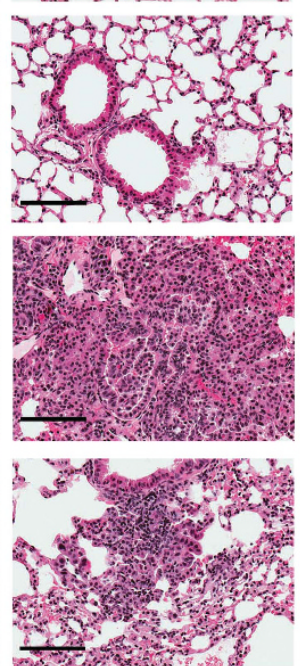

b
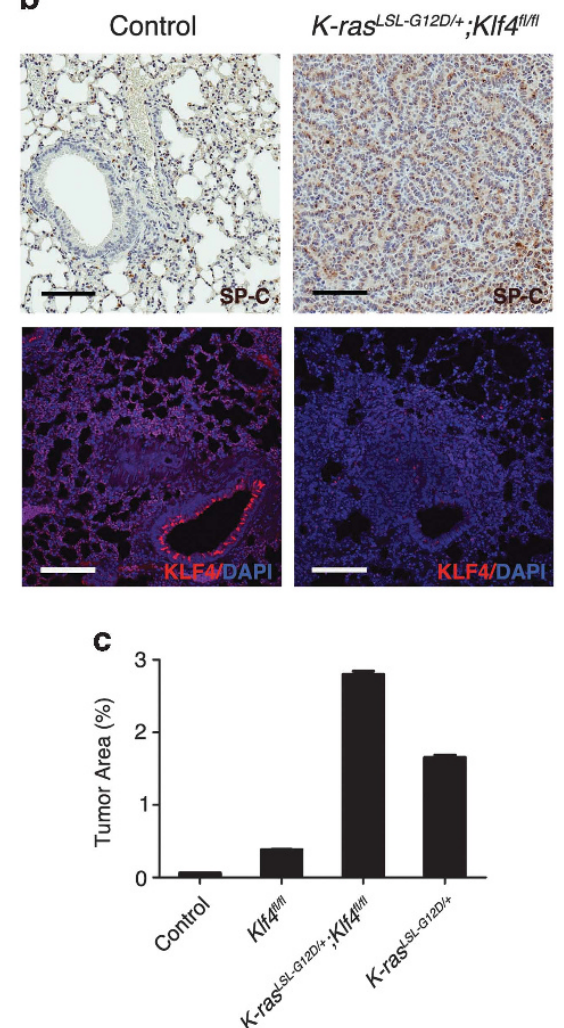

Figure 4 Klf4 deletion facilitates lung tumor formation and progression. (a) H\&E staining of the lung tissue from wild-type mice (control), the K-ras ${ }^{L S L-G 12 D /+}$ mice, KIff $4^{f / f t}$ mice and $K$-ras ${ }^{L S L-G 12 D /+} ; K / f 4^{f / / t / 1}$ mice by 8 weeks post infection. Scale bars from left to right: $2 \mathrm{~mm}$ and $100 \mu \mathrm{m}$. (b) IHC staining of SP-C (top) and immunofluorescent staining of KLF4 (red, bottom) showing that SP-C tends to increase in tumor tissue from $K-$ ras $^{L S L-G 12 D /+} ; K I f 4^{f / f t}$ mice than in normal tissue from wild-type mice. Scale bars: $100 \mu \mathrm{m}$. (c) Statistical analysis of the percentage of tumor area by 8 weeks post infection (based on H\&E staining). Data are represented as mean \pm S.D

hydroxamic (SAHA) and Trichostatin A (TSA), which are inhibitors of Class I, II and IV HDACs; and sodium butyrate $(\mathrm{NaBt})$ and valproic acid (VPA) (Figure $5 \mathrm{c}$ ), which are inhibitors of Class I and Ila HDACs. ${ }^{39}$ The cell proliferation assay also demonstrated that HDAC inhibitors repressed the growth of A549 and $\mathrm{H} 460$ cells (Figure $5 \mathrm{~d}$ ), which is consistent with their role in inducing KLF4 expression.

Studies have found that HDAC1 and KLF4 interact to regulate human myeloid leukemia cell proliferation. ${ }^{40}$ In order to study the correlation between HDACs and KLF4 in lung cancer cells, we did the luciferase reporter assays using GKLF-pGL2-Luciferase containing KLF4 promoter reporter. ${ }^{41}$ We found that HDAC3 has an inhibitory role on KLF4 promoter activity (Supplementary Figure 6).

\section{Discussion}

As an important regulator in cell differentiation and proliferation, KLF4 is essential in maintaining homeostasis and morphology in the lung. The Rosa-Cre ${ }^{+} ; K^{\prime / f 4^{f l / f l}}$ mouse model study provided evidence that KLF4 is functional in maintaining the number and differentiation pattern of SP-C-positive AT2 cells in the mouse lung during normal development (Figure 2). In addition, the $K$-ras ${ }^{L S L-G 12 D /+} ; K I f 4^{f / f t}$ mouse model study showed evidence that depletion of Klf4 facilitates lung tumor formation and progression through controlling the pattern of cell differentiation and proliferation (Figures 1 and 4). AT2 cells have been identified as stem cells in adult lung. ${ }^{20}$ The selfrenewal of AT2 cells can be induced by activated K-ras in vivo. ${ }^{21}$ Our data suggest that KIf4 deletion-induced AT2 cells are potential tumor-initiating cells leading to lung adenocarcinoma (Figures 2 and 4b). The tumor-suppressor role of KLF4 was further supported by the findings that KLF4 was significantly downregulated in human lung cancers (Figures $1 \mathrm{a}$ and $\mathrm{b}$ ).

The K-ras mouse models have been used to identify the origins of lung cancer. The earliest findings suggested that a rare population of cells located at the BADJ is CC10/SP-C double positive, which is capable of expanding in early $K$-ras mutant tumors induced by adenoviral-CMV-Cre, thus considered the origin of adenocarcinomas. ${ }^{32}$ Later studies in mice with activated $\mathrm{K}$-ras in $\mathrm{SP}_{-} \mathrm{C}^{+}$or $\mathrm{CC}^{-} 0^{+}$cells using conditional $\mathrm{Cre}^{\mathrm{ER}}$ knock-in alleles indicated that tumors arose only in the alveoli, although recombination occurred throughout the bronchioles, the BADJ and the bronchioalveolar stem cells, which are CC10/SP-C double-positive cells. ${ }^{21,31}$ We found that KLF4 deletion increased the number of AT2 cells and enhanced K-ras-mediated lung tumorigenesis (Figures $2 \mathrm{e}-\mathrm{g}$ and 4), consistent with the findings that AT2 cells are the major origin of lung cancer ${ }^{22}$ and that $\mathrm{K}$-ras regulates self-renewal of AT2 cells. ${ }^{21}$

Compared with lesions at 8 weeks post infection, more adenomas and adenocarcinomas arose at 16 weeks in K-ras ${ }^{L S L-G 12 D /+} ; K I f 4^{f / f t}$ mice, whereas only trace amount of 


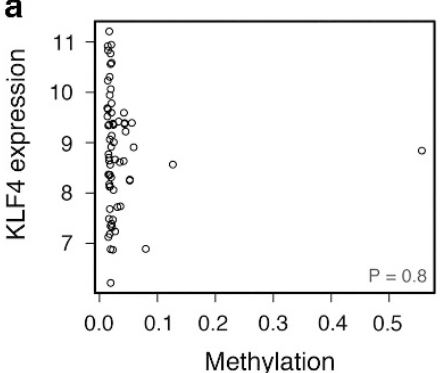

b

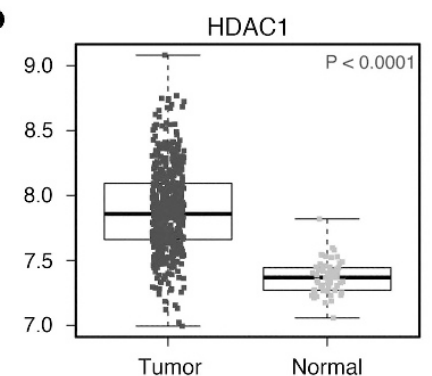

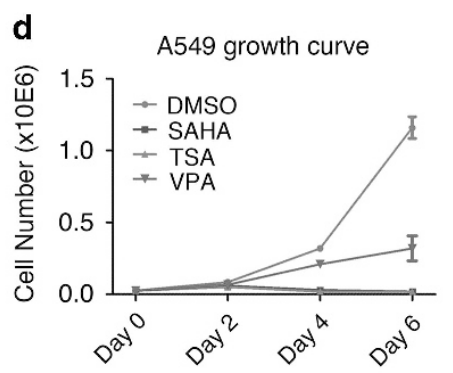

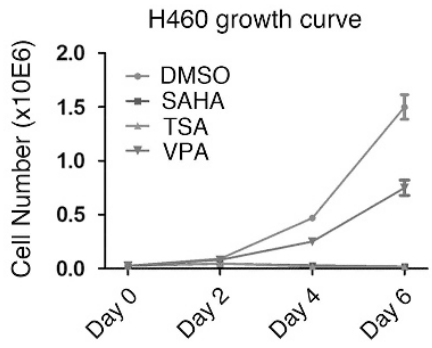

c

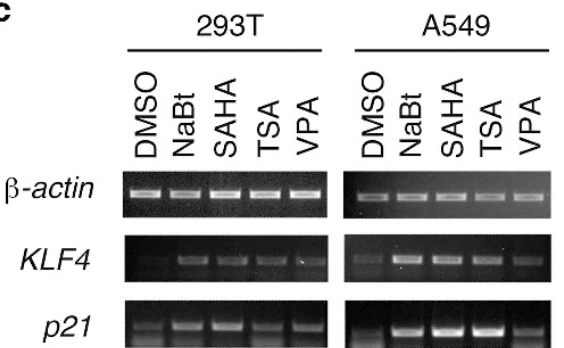

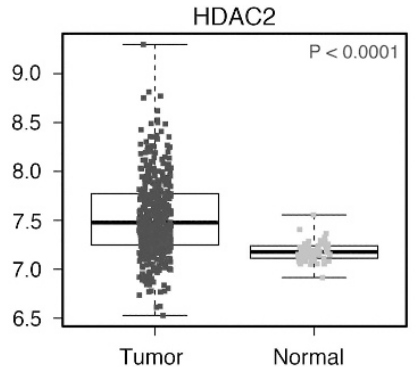

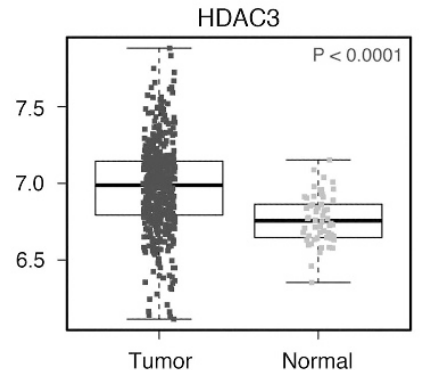

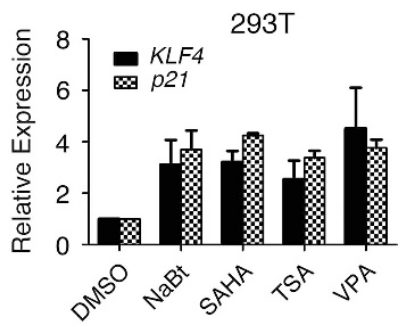

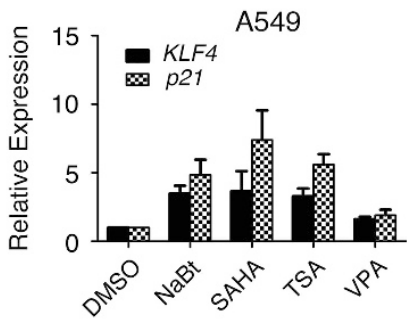

Figure 5 Multiple mechanisms of KLF4 downregulation in lung cancer. (a) Correlation between KLF4 expression and methylation in lung adenocarcinoma patients. Pearson's correlation coefficient was $-0.03(P=0.8,95 \%$ confidence interval -0.27 to 0.21$)$. (b) HDAC expression in normal lung and lung tumor tissues from TCGA lung adenocarcinoma samples. $P$ values were calculated based on linear mixed models. (c) Semi-quantitative RT-PCR (left) and real-time PCR (right) testing expression of KLF4 and target gene $p 21^{\text {cip } 1 \text { WAF1 }}$ in HEK293T and A549 cell lines when treated with HDAC inhibitors. Data are represented as mean \pm S.D. (d) Growth curve of A549 and H460 cell lines when treated with HDAC inhibitors

diffuse hyperplasia was found in KIff ${ }^{f / f I}$ and K-ras ${ }^{L S L-G 12 D /+}$ mouse lung (Supplementary Figure $7 \mathrm{a})$. The difference over time may be due to high dose of virus that we used $\left(2.5 \times 10^{7}\right.$ PFU), which may cause diffusion or absence of lesions in mouse lung. ${ }^{42}$ The average number of nodules of lung adenoma and adenocarcinoma in each lung lobe was greatly higher in $\mathrm{K}$-ras ${ }^{L S L-G 12 D /+} ; K^{\prime} / f 4^{f / f l}$ mice compared with control mice by 16 weeks (Supplementary Figure $7 b$ ).

KLF4 has multiple functions. It acts as a transcription factor by binding to the promoters of its target genes, such as cyclin $B 1$ and $p 21^{C i p 1 / W A F 1}$. It is a transcription activator as well as a repressor. In addition, it can bind other transcription factors and regulate their activity. KLF4 may regulate lung homeostasis and tumorigenesis through multiple mechanisms. For example, it has been reported that the stem cell marker Bmi1 have critical roles in lung tumorigenesis in $K$-ras ${ }^{G 12 D}$ mice. ${ }^{43}$ Bmi1 is critical for lung tumorigenesis and stem cell expansion. In the absence of oncogenic K-ras, Bmi1 deficiency led to impaired proliferation and self-renewal capacity of bronchiolalveolar stem cells. ${ }^{43}$ Our previous finding suggests that KLF4 inhibits Bmi1 expression in colorectal tumorigenesis. ${ }^{44}$ Using the mouse model from our current study, IHC staining of Bmi1 in the lung tissue showed that Bmi1 is overexpressed in tumor tissue in the K-ras ${ }^{L S L-G 12 D /+} ; K^{\prime} I f^{4 / / f I}$ mouse (Supplementary Figure 8). Both Bmi1 and AT2 cell markers were increased in the tumors, suggesting that KLF4 deletion induced tumorinitiating cells during lung tumorigenesis.

KLF4 was first defined as required for establishing the barrier function of the skin. ${ }^{45}$ Studies have shown that KLF4 has a role in mediating proinflammatory signaling in macrophages, ${ }^{46,47}$ and it regulates anti-inflammatory cytokines such as interleukin-10. ${ }^{48}$ Chronic lung inflammation has been associated with tumorigenesis in the lung. As one of the most common genetic changes in lung cancer, $K$-ras activation induces a robust inflammatory response. ${ }^{49}$ Based on observations from H\&E staining, Adenovirus-Cre administration induced certain areas of inflammatory infiltrates in the lung (Figure 4a). Myeloperoxidase (MPO) is a marker for neutrophils, which are important members of the innate immune system, and which are highly recruited to the site of inflammation during the immune response. We stained the lung tissues for MPO and compared the percentage of $\mathrm{MPO}^{+}$ 
cells. Although adenoviral infection could induce inflammation, which is associated with bronchiolar hyperplasia, ${ }^{22}$ Klf4 deletion and $K$-ras mutation in combination had greatly enhanced the inflammatory response (Supplementary Figure 9). Moreover, higher intensity of MPO staining cells in K-ras ${ }^{L S L-G 12 D /+} ; K_{I f 4^{f l / f l}}$ mice are mainly localized at the tumor regions (Supplementary Figures $9 \mathrm{~g}$ and $\mathrm{h}$ ). The above data indicate that KIf4 depletion triggered lung inflammation, which may contribute to lung tumorigenesis in the mice. Our findings suggest that KLF4 has an important role in protecting lung tissue from inflammation and inflammation-associated tumorigenesis.

Our study also demonstrated that mutations and methylation are not the major mechanisms that cause the downregulation of KLF4 in lung cancer (Figures 1a and $5 \mathrm{a}$, Supplementary Figures $1 \mathrm{a}, \mathrm{b}$ and $5 \mathrm{a}$ ), whereas analysis of HDACs in lung cancer database and lung cancer cells revealed an important mechanism by which KLF4 is regulated in lung tumorigenesis (Figure 5 and Supplementary Figures 5 and 6). HDACs are grouped in four families. ${ }^{50}$ Among these, type III HDACs are known as sirtuins. The expression profiles of sirtuins in the lung cancer patients have not been analyzed in this study. HDAC6 belongs to group Ilb along with $\mathrm{HDAC} 10$, and it has unique structural and functional features. ${ }^{51,52}$ We also treated cells with an HDAC6 inhibitor, $\mathrm{ACY}-1215,{ }^{53}$ but found no change in KLF4 expression (not shown). It is very interesting that all four members of Type I HDACs were significantly increased in human lung cancers (Figure 5b and Supplementary Figure 5b). HDAC3 has an inhibitory role on KLF4 promoter activity in HEK293T cells. In A549 cells, the effect was not significant (Supplementary Figure 6). This could be due to the high level of HDAC3 expression in lung cancer cells or because of the redundant function of other HDACs. Previous studies found that KLF4 recruits HDAC3 to the cyclinB1 promoter and that KLF4 synergized with HDAC3 in repressing the cyclinB1 promoter activity. ${ }^{28}$ This could be another mechanism that Class I HDAC inhibitors can regulate KLF4 and its target genes. The studies of HDAC inhibitors (Figures $5 \mathrm{c}$ and $\mathrm{d}$ ) indicate that HDAC inhibitors inhibit lung cancer cell growth at least partially through inducing KLF4 expression. As discovered in many other types of malignancy, ${ }^{54,55}$ it is of potential importance to develop specific type I HDAC inhibitors targeting lung cancer-initiating cells.

In addition to KLF4, other members of KLF family can be altered in lung cancers ${ }^{29}$ (Supplementary Figure 2). It is of great interest to further study the function of other KLF transcription factors in lung tumor initiation and progression.

\footnotetext{
Materials and Methods

Animal studies. Mouse experiments were performed under the approval by the Institutional Animal Care and Use Committees of the University of Kentucky (20090604). Mice of Cre-inducible Klft//fll were crossed with mice of the Cre-inducible K-ras ${ }^{L S L-G 12 D /+}$ strain. All mice used for experiments were on C57BL/6 genetic background. Mice were genotyped by PCR using genome DNA samples from mice tails. Adenovirus Ad5CMVCre-eGFP was from Gene Transfer Vector Core at the University of lowa. Adenovirus was used to induce Klf4 deletion and/or K-ras mutation by intranasal infection technique. ${ }^{56}$ Mice were killed 8-16 weeks after the virus infection for analysis. For the Kl/4 $4^{f / f l}$, Ad5CMVCre-eGFP induces the 'flox' of critical exons of Klf4 gene. And for K-ras ${ }^{L S L-G 12 D /+}$, Ad5CMVCre-eGFP induces the 'flox' of the 'stop' element ('LSL'), to activate the mutant K-ras ${ }^{G 12 D}$ expression.
}

Cell culture and proliferation assay. Human lung cancer cell lines A549 and $\mathrm{H} 460$ were cultured in RPMI 1640 containing $10 \%$ fetal bovine serum and $1 \%$ penicillin/streptomycin. For proliferation assay, cells were plated at $\sim 2.5 \times 10^{4}$ cells per well in 12-well plates and counted at appropriate times using the cell viability analyzer (Beckman Coulter, Indianapolis, IN, USA, Vi-Cell XR). For proliferation assay in Figure 1c, cells were infected with vector-carrying or KLF4-carrying lentivirus, and equal numbers of cells were seeded to 12 -well plates $18 \mathrm{~h}$ post infection, thus counted as day 0 .

Western blotting. Cells were lysed in the appropriate volume of lysis buffer (50 mM HEPES, $100 \mathrm{mM} \mathrm{NaCl}, 2 \mathrm{mM}$ EDTA, $1 \%$ glycerol, $50 \mathrm{mM} \mathrm{NaF}, 1 \mathrm{mM}$ $\mathrm{Na}_{3} \mathrm{VO}_{4}, 1 \%$ Triton $\mathrm{X}-100$, with protease inhibitors). The following antibodies were used: mouse anti-GAPDH (GeneTex, Irvine, CA, USA, GT239), rabbit-anti-KLF4 ${ }^{15}$ and Bmi1 (Epitomics, Burlingame, CA, USA, S2983).

RT-PCR and real-time PCR. A549 and H460 cells were plated at $~ 2 \times 10^{5}$ cells per well in a six-well plate to be infected by vector-carrying or KLF4-carrying lentivirus. HEK293T and A549 cells were treated with lentivirus or the following molecules: DMSO (control, 1:1000 in volume); sodium butyrate $(5 \mathrm{mM})$; suberoylanilide hydroxamic $(10 \mu \mathrm{M})$; Trichostatin A $(1 \mu \mathrm{M})$; VPA (1 mM). After $48 \mathrm{~h}$ of incubation, RNA was extracted using the RNeasy kit (Qiagen, Valencia, CA, USA). Reverse transcriptase PCR (RT-PCR) was performed as described previously. ${ }^{15}$

Real-time RT-PCR reactions were carried out using SYBR Green PCR master mix reagents (Thermo, Waltham, MA, USA) on the ABI StepOnePlus Real-Time PCR System (Applied Biosystems, Life Technologies, Carlsbad, CA, USA). Thermal cycling was conducted at $95^{\circ} \mathrm{C}$ for $10 \mathrm{~min}$, followed by 40 cycles of amplification at $95^{\circ} \mathrm{C}$ for $15 \mathrm{~s}$ and $60^{\circ} \mathrm{C}$ for $1 \mathrm{~min}$, then the melt curve: $95^{\circ} \mathrm{C}$ for $15 \mathrm{~s}, 60^{\circ} \mathrm{C}$ for $1 \mathrm{~min}$ and $95^{\circ} \mathrm{C}$ for $15 \mathrm{~s}$. The relative quantification of gene expression for each sample was analyzed by the $\triangle \mathrm{Ct}$ method. The following primers were used: $\beta$-actin, $5^{\prime}$-CAA CCGCGAGAAGATGAC-3' and 5'-AGGAAGGTGGAAGAGTG-3'; $21^{\text {Cip1 } 1 \text { WAF1: }}$ 5'-CGACTGTGATGCGCTAATGG-3' and 5'-AGAAGATCAGCCGGCGTTTG-3'; cyclin B1, 5'-GCAGCACCTGGCTAAGAATG-3' and 5'-GCCACAGCCTTGGC TAAATC-3'; KLF4, 5'-AGAGGAGCCCAAGCCAAAG-3' and 5'-CGTCCCAGT CACAGTGGTAAG-3'.

H\&E staining and IHC staining. H\&E staining was performed based on standard protocol by the Markey Cancer Center Biospecimen and Tissue Procurement Shared Resource Facility at the University of Kentucky. For IHC staining, the following antibodies were used: rabbit anti-KLF4, ${ }^{15}$ goat anti-CC10 (T-18, Santa Cruz, Dallas, TX, USA, sc-9772), rabbit anti-SP-C (FL-197, Santa Cruz, sc-13979), mouse anti-FoxJ1 (eBioscience, San Diego, CA, USA, 14-9965), mouse anti-Cav1 (7C8, NovusBio, Littleton, CO, USA, NB100-615SS), rabbit antiBmi1 (Epitomics, Burlingame, CA, USA, S2983), rabbit anti-Myeloperoxidase (Acris, San Diego, CA, USA, AP23272PU-N). Slides were scanned by the Aperio ScanScope XT and analyzed by nuclearv 9 algorithm in the Aperio Analysis software (Leica Biosystems, Buffalo Grove, IL, USA).

Immunofluorescent staining. Deparaffinized slides were incubated with antigen retrieval buffer and blocking solution with $5 \%$ goat serum in PBST for 20 and $10 \mathrm{~min}$, respectively. Slides then were incubated with primary antibody overnight at $4^{\circ} \mathrm{C}$, followed by secondary antibody for $1 \mathrm{~h}$ at room temperature. RhodamineTyramide and FITC-Tyramide were used to amplify signals from the secondary antibodies, according to the previous protocol. ${ }^{57}$ Primary antibodies used include rabbit anti-KLF4 ${ }^{15}$ and rabbit anti-SP-C (FL-197, Santa Cruz, sc-13979). The secondary antibody used was goat anti-rabbit-HRP (Jackson, West Grove, PA, USA, 111-035003). Nuclei were stained by DAPI (Sigma, St. Louis, MO, USA). The glass slides were mounted with coverslips, viewed and photographed with an Olympus FW1000 confocal microscope.

Statistical analysis. Normalized RNAseq(V2) gene expression data were downloaded from TCGA and $\log _{2}$-transformed. The following numbers of patients were included: 488 lung adenocarcinoma versus 58 normal specimens; 501 lung small-cell carcinoma versus 51 normal specimens. The expressions of KLF4 and HDACs in tumor and normal samples were compared based on linear mixed models, which accounted for the correlation between tumor and normal samples from the same individual. DNA methylation data (beta values) from 65 lung adenocarcinoma specimens and 65 lung small-cell carcinoma specimens were 
downloaded from TCGA. Pearson's correlation coefficient was used to assess the correlation between KLF4 mRNA expression and methylation in lung adenocarcinoma specimens and in lung small-cell carcinoma specimens. Statistical significance was defined as $P<0.05$.

\section{Conflict of Interest}

The authors declare no conflict of interest.

Acknowledgements. We thank Garretson Epperly for technical support in image scan and analysis and the Markey Biospecimen and Tissue Procurement Shared Resource Facility for the tissue embedding, sectioning and H\&E staining. And we thank Vivek M Rangnekar for the BEAS-2B cell line, and Vincent W Yang for the GKLF-pGL2-Luciferase reporter. The University of Kentucky Biospecimen and Tissue Procurement and Biostatistics and Bioinformatics Shared Resource Facilities are supported by the Markey Cancer Center (P30 CA177558). CL was supported by R01 CA172379 from the NIH.

1. Siegel R, Ma J, Zou Z, Jemal A. Cancer statistics, 2014. CA Cancer J Clin 2014; 64: 9-29.

2. Sun S, Schiller JH, Gazdar AF. Lung cancer in never smokers-a different disease. Nat Rev Cancer 2007; 7: 778-790.

3. Westra WH, Slebos RJ, Offerhaus GJ, Goodman SN, Evers SG, Kensler TW et al. K-ras oncogene activation in lung adenocarcinomas from former smokers. Evidence that K-ras mutations are an early and irreversible event in the development of adenocarcinoma of the lung. Cancer 1993; 72: 432-438.

4. Johnson L, Mercer K, Greenbaum D, Bronson RT, Crowley D, Tuveson DA et al. Somatic activation of the K-ras oncogene causes early onset lung cancer in mice. Nature 2001; 410 1111-1116.

5. Shields JM, Christy RJ, Yang VW. Identification and characterization of a gene encoding a gut-enriched Kruppel-like factor expressed during growth arrest. J Biol Chem 1996; 271 20009-20017.

6. Garrett-Sinha LA, Eberspaecher H, Seldin MF, de Crombrugghe B. A gene for a nove zinc-finger protein expressed in differentiated epithelial cells and transiently in certain mesenchymal cells. J Biol Chem 1996; 271: 31384-31390.

7. Panigada M, Porcellini S, Sutti F, Doneda L, Pozzoli O, Consalez GG et al. GKLF in thymus epithelium as a developmentally regulated element of thymocyte-stroma cross-talk. Mech Dev 1999; 81: 103-113.

8. Chiambaretta F, De Graeve F, Turet G, Marceau G, Gain P, Dastugue B et al. Cell and tissue specific expression of human Kruppel-like transcription factors in human ocular surface. Mol Vis 2004; 10: 901-909.

9. Cullingford TE, Butler MJ, Marshall AK, Tham el L, Sugden PH, Clerk A. Differential regulation of Kruppel-like factor family transcription factor expression in neonatal rat cardiac myocytes: effects of endothelin-1, oxidative stress and cytokines. Biochim Biophys Acta 2008; 1783: 1229-1236.

10. Fruman DA, Ferl GZ, An SS, Donahue AC, Satterthwaite AB, Witte ON. Phosphoinositide 3-kinase and Bruton's tyrosine kinase regulate overlapping sets of genes in B lymphocytes. Proc Natl Acad Sci USA 2002; 99: 359-364.

11. Ton-That H, Kaestner KH, Shields JM, Mahatanankoon CS, Yang VW. Expression of the gut-enriched Kruppel-like factor gene during development and intestinal tumorigenesis. FEBS Lett 1997; 419: 239-243.

12. Katz JP, Perreault N, Goldstein BG, Lee CS, Labosky PA, Yang VW et al. The zinc-finger transcription factor Klf4 is required for terminal differentiation of goblet cells in the colon. Development 2002; 129: 2619-2628.

13. Jean JC, George E, Kaestner KH, Brown LA, Spira A, Joyce-Brady M. Transcription factor $\mathrm{Klf} 4$, induced in the lung by oxygen at birth, regulates perinatal fibroblast and myofibroblast differentiation. PLoS One 2013; 8: e54806.

14. Zhao W, Hisamuddin IM, Nandan MO, Babbin BA, Lamb NE, Yang VW. Identification of Kruppel-like factor 4 as a potential tumor suppressor gene in colorectal cancer. Oncogene 2004; 23: 395-402.

15. Zhang W, Chen X, Kato Y, Evans PM, Yuan S, Yang J et al. Novel cross talk of Kruppel-like factor 4 and beta-catenin regulates normal intestinal homeostasis and tumor repression. Molecular and cellular biology 2006; 26: 2055-2064.

16. Ghaleb AM, McConnell BB, Nandan MO, Katz JP, Kaestner KH, Yang VW. Haploinsufficiency of Kruppel-like factor 4 promotes adenomatous polyposis coli dependent intestinal tumorigenesis. Cancer Res 2007; 67: 7147-7154.

17. Takahashi K, Tanabe K, Ohnuki M, Narita M, Ichisaka T, Tomoda K et al. Induction of pluripotent stem cells from adult human fibroblasts by defined factors. Cell 2007; 131: 861-872.

18. Takahashi K, Yamanaka S. Induction of pluripotent stem cells from mouse embryonic and adult fibroblast cultures by defined factors. Cell 2006; 126: 663-676.

19. Hu W, Hofstetter WL, Li H, Zhou Y, He Y, Pataer A et al. Putative tumor-suppressive function of Kruppel-like factor 4 in primary lung carcinoma. Clin Cancer Res 2009; 15: 5688-5695.
20. Barkauskas CE, Cronce MJ, Rackley CR, Bowie EJ, Keene DR, Stripp BR et al. Type 2 alveolar cells are stem cells in adult lung. The Journal of clinical investigation 2013; 123: 3025-3036.

21. Desai TJ, Brownfield DG, Krasnow MA. Alveolar progenitor and stem cells in lung development, renewal and cancer. Nature 2014; 507: 190-194.

22. Mainardi S, Mijimolle N, Francoz S, Vicente-Duenas C, Sanchez-Garcia I, Barbacid M. Identification of cancer initiating cells in K-Ras driven lung adenocarcinoma. Proc Natl Acad Sci USA 2014; 111: 255-260.

23. Sutherland KD, Song JY, Kwon MC, Proost N, Zevenhoven J, Berns A. Multiple cells-oforigin of mutant K-Ras-induced mouse lung adenocarcinoma. Proc Natl Acad Sci USA 2014; 111: $4952-4957$.

24. Ghaleb AM, Yang VW. The Pathobiology of Kruppel-like Factors in Colorectal Cancer. Current colorectal cancer reports 2008; 4: 59-64.

25. Ghaleb AM, Nandan MO, Chanchevalap S, Dalton WB, Hisamuddin IM, Yang VW. Kruppel-like factors 4 and 5 : the yin and yang regulators of cellular proliferation. Cell Research 2005; 15: 92-96.

26. Reuss DE, Piro RM, Jones DT, Simon M, Ketter R, Kool M et al. Secretory meningiomas are defined by combined KLF4 K409Q and TRAF7 mutations. Acta Neuropathol 2013; 125 : 351-358.

27. Clark VE, Erson-Omay EZ, Serin A, Yin J, Cotney J, Ozduman K et al. Genomic analysis of non-NF2 meningiomas reveals mutations in TRAF7, KLF4, AKT1, and SMO. Science 2013; 339: 1077-1080.

28. Evans PM, Zhang W, Chen X, Yang J, Bhakat KK, Liu C. Kruppel-like factor 4 is acetylated by p300 and regulates gene transcription via modulation of histone acetylation. J Biol Chem 2007; 282: 33994-34002.

29. Tetreault MP, Yang Y, Katz JP. Kruppel-like factors in cancer. Nat Rev Cancer 2013; 13: 701-713.

30. Zambrowicz BP, Imamoto A, Fiering S, Herzenberg LA, Kerr WG, Soriano P. Disruption of overlapping transcripts in the ROSA beta geo 26 gene trap strain leads to widespread expression of beta-galactosidase in mouse embryos and hematopoietic cells. Proc Natl Acad Sci USA 1997; 94: 3789-3794.

31. Xu X, Rock JR, Lu Y, Futtner C, Schwab B, Guinney J et al. Evidence for type II cells as cells of origin of K-Ras-induced distal lung adenocarcinoma. Proc Natl Acad Sci USA 2012; 109: 4910-4915.

32. Kim CF, Jackson EL, Woolfenden AE, Lawrence S, Babar I, Vogel S et al. Identification of bronchioalveolar stem cells in normal lung and lung cancer. Cell 2005; 121: 823-835.

33. Malkinson AM. Genetic studies on lung tumor susceptibility and histogenesis in mice. Environ Health Perspect 1991; 93: 149-159.

34. Rowbotham SP, Kim CF. Diverse cells at the origin of lung adenocarcinoma. Proc Natl Acad Sci USA 2014; 111: 4745-4746.

35. Abrigo M, Alvarez R, Paparella ML, Calb D, Bal de Kier Joffe E, Gutkind JS et al. Impairing squamous differentiation by Klf4 deletion is sufficient to initiate tongue carcinoma development upon K-Ras activation in mice. Carcinogenesis 2013; 35: 662-669.

36. Evans PM, Chen X, Zhang W, Liu C. KLF4 interacts with beta-catenin/TCF4 and blocks p300/CBP recruitment by beta-catenin. Molecular and cellular biology 2010; 30: 372-381.

37. Shahbazian MD, Grunstein M. Functions of site-specific histone acetylation and deacetylation. Annu Rev Biochem 2007; 76: 75-100.

38. Barneda-Zahonero B, Parra M. Histone deacetylases and cancer. Mol Oncol 2012; 6: 579-589.

39. Carew JS, Giles FJ, Nawrocki ST. Histone deacetylase inhibitors: mechanisms of cell death and promise in combination cancer therapy. Cancer Letters 2008; 269: 7-17.

40. Huang Y, Chen J, Lu C, Han J, Wang G, Song C et al. HDAC1 and Klf4 interplay critically regulates human myeloid leukemia cell proliferation. Cell death \& disease 2014; 5: e1491.

41. Mahatan CS, Kaestner KH, Geiman DE, Yang VW. Characterization of the structure and regulation of the murine gene encoding gut-enriched Kruppel-like factor (Kruppel-like factor 4). Nucleic acids research 1999; 27: 4562-4569.

42. Jackson EL, Willis N, Mercer K, Bronson RT, Crowley D, Montoya R et al. Analysis of lung tumor initiation and progression using conditional expression of oncogenic K-ras. Genes Dev 2001; 15: 3243-3248.

43. Dovey JS, Zacharek SJ, Kim CF, Lees JA. Bmi1 is critical for lung tumorigenesis and bronchioalveolar stem cell expansion. Proc Natl Acad Sci USA 2008; 105 : 11857-11862.

44. Yu T, Chen X, Zhang W, Colon D, Shi J, Napier D et al. Regulation of the potential marker for intestinal cells, Bmi1, by beta-catenin and the zinc finger protein KLF4: implications for colon cancer. J Biol Chem 2012; 287: 3760-3768.

45. Segre JA, Bauer C, Fuchs E. Klf4 is a transcription factor required for establishing the barrier function of the skin. Nat Genet 1999; 22: 356-360.

46. Feinberg MW, Cao Z, Wara AK, Lebedeva MA, Senbanerjee S, Jain MK. Kruppel-like factor 4 is a mediator of proinflammatory signaling in macrophages. J Biol Chem 2005; 280: 38247-38258.

47. Liu J, Zhang H, Liu Y, Wang K, Feng Y, Liu M et al. KLF4 regulates the expression of interleukin-10 in RAW264.7 macrophages. Biochem Biophys Res Commun 2007; 362 : 575-581.

48. Zahlten J, Steinicke R, Bertrams W, Hocke AC, Scharf S, Schmeck B et al. TLR9- and Src-dependent expression of Krueppel-like factor 4 controls interleukin-10 expression in pneumonia. Eur Respir J 2013; 41: 384-391. 
49. Ji H, Houghton AM, Mariani TJ, Perera S, Kim CB, Padera R et al. K-ras activation generates an inflammatory response in lung tumors. Oncogene 2006; 25: 2105-2112.

50. Yang XJ, Seto $E$. The Rpd3/Hda1 family of lysine deacetylases: from bacteria and yeast to mice and men. Nat Rev Mol Cell Biol 2008; 9: 206-218.

51. Boyault C, Sadoul K, Pabion M, Khochbin S. HDAC6, at the crossroads between cytoskeleton and cell signaling by acetylation and ubiquitination. Oncogene 2007; 26: 5468-5476.

52. Simoes-Pires C, Zwick V, Nurisso A, Schenker E, Carrupt PA, Cuendet M. HDAC6 as a target for neurodegenerative diseases: what makes it different from the other HDACs? Mol Neurodegener 2013; 8: 7.

53. Santo L, Hideshima T, Kung AL, Tseng JC, Tamang D, Yang M et al. Preclinical activity, pharmacodynamic, and pharmacokinetic properties of a selective HDAC6 inhibitor,
ACY-1215, in combination with bortezomib in multiple myeloma. Blood 2012; 119: 2579-2589.

54. Kee HJ, Kwon JS, Shin S, Ahn Y, Jeong MH, Kook H. Trichostatin A prevents neointimal hyperplasia via activation of Kruppel like factor 4. Vascul Pharmacol 2011; 55: 127-134.

55. Kee HJ, Kook H. Kruppel-like factor 4 mediates histone deacetylase inhibitor-induced prevention of cardiac hypertrophy. J Mol Cell Cardiol 2009; 47: 770-780.

56. DuPage M, Dooley AL, Jacks T. Conditional mouse lung cancer models using adenoviral or lentiviral delivery of Cre recombinase. Nat Protoc 2009; 4: 1064-1072.

57. Hopman AH, Ramaekers FC, Speel EJ. Rapid synthesis of biotin-, digoxigenin-, trinitrophenyl-, and fluorochrome-labeled tyramides and their application for In situ hybridization using CARD amplification. J Histochem Cytochem 1998; 46: 771-777.

Supplementary Information accompanies this paper on Cell Death and Differentiation website (http://www.nature.com/cdd) 